\title{
LA AGENDA OLÍMPICA 2020: DESAFÍOS Y OPORTUNIDADES DE LAS 40 RECOMENDACIONES PARA LA SOSTENIBILIDAD Y CREDIBILIDAD DEL MOVIMIENTO OLÍMPICO
}

\author{
Carlos Eduardo Villegas Estrada \\ Miembro de número de la Academia Olímpica Colombiana \\ Comité Olímpico Colombiano \\ carlosevillegas@hotmail.com
}

Fecha de recepción: Mayo 2016

Fecha de aceptación: Septiembre 2016

http://dx.doi.org/10.15366/citius2016.9.2.001

\section{Resumen:}

Este artículo hace un arqueo de las 40 recomendaciones contenidas en la denominada "Agenda Olímpica 2020, la estratégica hoja de ruta para el futuro del Movimiento Olímpico" y las analiza en una perspectiva de sostenibilidad, credibilidad y juventud, enmarcada en la profunda crisis de confianza por la que atraviesa el deporte mundial. La Agenda propone una apertura del Movimiento Olímpico para jugar un papel más protagónico en una sociedad globalizada y regida por la era digital. A través de una opinión crítica se identifican los desafíos y las oportunidades que la Agenda ofrece, y se pretende establecer hasta qué punto se trata de una profunda reforma o de una simple evolución del deporte inherente al proceso civilizador cuyo origen se remontan al antiguo imperio griego.

Palabras clave: Agenda Olímpica 2020, deporte, juventud, apertura, credibilidad, sostenibilidad, era digital.

Title: THE OLYMPIC AGENDA 2020: CHALLENGES AND OPPORTUNITIES OF THE 40 RECOMENDATIONS FOR THE SUTAINABILITY AND CREDIBILITY OF THE OLYMPIC MOVEMENT.

\section{Abstract:}

This paper pans the 40 recommendations contained in the so-called "Olympic Agenda 2020 a strategic roadmap for the future of the Olympic Movement". An analysis is made in regards to the calls to sustainability, credibility and youth, that respond to the deep crisis of confidence affecting the world of sport. The Agenda aims at fostering a more visible role of the Olympic Movement in a globalized digitalized society governed by the digital age. Through a critical lens, the challenges and opportunities offered by that Agenda 2020 are commented. The purpose is to establish the extent to which this is a deep reform or a simple evolution of sport inherent to the civilizing process whose origins date back to the ancient Greek empire.

Key words: Olympic Agenda 2020, sport, youth, openness, credibility, sustainability, digital era.

\section{Introducción}

La industria o sector del deporte atraviesa por una profunda crisis de confianza y credibilidad originada en escándalos de corrupción que terminaron con la separación de sus cargos de dos omnipotentes dirigentes deportivos, el suizo Joseph Blatter, presidente de la Federación Internacional de Asociaciones de Fútbol -FIFA- y del senegalés Lamine Diack, miembro honorario del Comité Olímpico Internacional -COI- y presidente honorario de la Asociación Internacional de Federaciones Atléticas -IAAF-; algo impensado hasta hace unos pocos años. 
Estos casos, sumados a las recientes denuncias de amaño de partidos y manipulación de resultados en el tenis profesional ponen una enorme presión en el COI como organización que reconoce a las más importantes federaciones deportivas internacionales pertenecientes al programa de los Juegos Olímpicos. Para un análisis profundo y actualizado sobre la corrupción en el deporte, véase Global Corruption Report: Sport, publicado por Transparency International en 2016 (Transparency International, 2016).

La Agenda Olímpica 2020, una hoja de ruta para el futuro del Movimiento Olímpico, aprobada en esa histórica Sesión del COI realizada en el Principado de Mónaco entre el 7 y el 9 de diciembre de 2014, no podría haber llegado en un momento más oportuno. El gestor de esta audaz iniciativa se llama Thomas Bach, de nacionalidad alemana, el noveno Presidente COI en sus 120 años de historia, pero el primero en haber conquistado una medalla de oro Olímpica como atleta, en la prueba de esgrima de florete por equipos, durante los Juegos de la XXI Olimpiada, celebrados en Montreal en 1976.

A diferencia de sus antecesores, el presidente Bach no convocó un Congreso Olímpico para repensar el Movimiento Olímpico, una opción prevista en los Estatutos del COI, conocidos como la Carta Olímpica, sino que se la jugó con una agenda propia alrededor de la cual logró el consenso de la mayoría de las partes interesadas o stakeholders. Más sorprendente aún, resulta el hecho de que esta propuesta de cambio la realice, quizá, en el momento de mayor esplendor del $\mathrm{COI}$ en su historia. La historia se encargará de dar cuenta si estamos frente al inicio de una nueva era del Movimiento Olímpico y qué tan efectivas y oportunas resultaron estas recomendaciones lideradas por el Presidente Bach.

\section{El éxito: la mejor razón para el cambio ¿Simple evolución o cambio real?}

Durante el discurso de instalación de la $127^{\mathrm{a}}$ Sesión en el Principado de Mónaco, refiriéndose a la Agenda 2020, el Presidente Bach propuso emprender una reforma, en medio del éxito que representaron los Juegos Olímpicos de Londres 2012 y Sochi 2014, seguidos por millonarias audiencias alrededor del mundo, sumados a una solidez y estabilidad financiera, sin precedentes en la centenaria historia del COI. "El éxito, la mejor razón para el cambio [...], cambiar o ser cambiados [...], el éxito del ayer no significa nada para el hoy" fueron algunas de las reflexiones de Bach (International Olympic Committee, 2014, p. 4).

La solvencia económica del COI se refleja en ingresos para el cuatrienio 2013-2016 de más de 5.5 billones de dólares americanos, una utilidad neta de 67 millones en 2014, y un patrimonio superior a los 2.8 billones. Estas cifras son un indicador del potencial del deporte, en general, y del Movimiento Olímpico en particular, para liderar procesos sociales o de civilización, para ponerlo en palabras de Elias, "consciente de que el conocimiento acerca del deporte lo era también de la sociedad" (Elias \& Dunning, 1995, p. 31).

Los Juegos Olímpicos de Londres 2012 el evento más visto en la historia; por ejemplo, el 90\% de los habitantes del Reino Unido vieron las transmisiones de la BBC con 52 millones de personas sintonizándolos mínimo 15 minutos; mientras que, de acuerdo con la NBC, más de 219 millones de norteamericanos siguieron los Juegos a través de sus canales (International Olympic Committee, 2013). Por su parte, los Juegos de Sochi 2014, los últimos celebrados, marcaron el lanzamiento de la aplicación OVP (Olympic Video Player) desarrollada por el COI para transmitir vía streaming y por demanda, videos de todos los eventos de los Juegos; que resultó en el consumo de más de dos millones de horas de transmisión de audios y videos por vía digital, lo que superó, incluso, el número de horas de transmisión por televisión convencional (International Olympic Committee, 2015). 
La fortaleza y estabilidad del COI se reflejan también en el proyecto de construcción de sus nuevas oficinas generales en Lausana, con el propósito de consolidar todas las dependencias y los cerca de 600 colaboradores del COI bajo un mismo techo, de maximizar sus operaciones y de atender la creciente demanda de sus programas de una manera más eficiente. El proyecto diseñado por la oficina de arquitectos holandesa $3 \mathrm{XN}$, tendrá un área de $24.000 \mathrm{mts}^{2}$ con una inversión de 139 millones de dólares (Butler, 2016). Con la nueva construcción se busca reducir, a largo plazo, los costos de operación, con una propuesta sostenible y generosa con el medio ambiente, tal y como lo recomienda la Agenda 2020.

\section{Del Congreso Olímpico a la Agenda Olímpica}

El afán reformista de Thomas Bach y, quizá, un deseo de diferenciar su mandato del de sus antecesores, lo indujo a cambiar la metodología para impulsar su propia reforma. Tan solo dos meses después de su posesión como Presidente, presentó ante el Comité Ejecutivo del COI su idea de reforma, rompiendo con la tradición centenaria de realizar profundos cambios a través de los Congresos Olímpicos. ¿Acabó la Agenda 2020 con los Congresos Olímpicos? Pareciera que, por lo menos durante el mandato de Bach, difícilmente se realizará.

Su antecesor, Jacques Rogge, por ejemplo, convocó el XIII Congreso Olímpico en 2009, es decir, tras 8 años como Presidente del COI. Por su parte, Juan Antonio Samaranch, quien ostentó el poder del COI por 21 años, convocó durante su mandato dos Congresos Olímpicos, uno en 1981 y otro en 1994, denominado el Congreso de la Unidad o del Centenario, por conmemorarse los 100 años de la creación del Comité Olímpico Internacional. En los 122 años de historia del COI se han convocado 13 Congresos Olímpicos, siendo el primero el de París en 1894. Presidido por el barón Pierre de Coubertin, fue justamente durante este Congreso de París donde, no solo se acordó crear el Comité Olímpico Internacional que sería presidido por el Griego Demetrio Vikelas, sino que se restauraron los Juegos Olímpicos (Villegas Estrada, 2010).

Las recomendaciones de la Agenda Olímpica 2020 son el resultado de un año de diálogo participativo donde concurrieron, además de la administración y los miembros del COI, las Federaciones Deportivas Internacionales, los Comités Olímpicos Nacionales, las Organizaciones Deportivas reconocidas, los patrocinadores del COI, grupos de académicos y de la sociedad civil en general. Durante el proceso se recibieron 1.200 ideas provenientes de 270 contribuidores y 43.500 e-mails con todo tipo de aportes (International Olympic Committee, 2014, p. 1).

\section{Un rompecabezas de 40 piezas, 5 grupos y 3 ejes}

La iniciativa de una reforma fue considerada por primera vez en un "retiro" del Comité Ejecutivo del COI, realizado en Montreux en diciembre de 2013 y posteriormente llevado al pleno de la $126^{a}$ Sesión del COI realizada en Sochi en febrero de 2014. Se conformaron, 14 grupos de trabajo que partieron de 25 temas centrales que resultaron en 40 recomendaciones presentadas, inicialmente al Comité Ejecutivo en octubre de 2014 y finalmente a la histórica $127^{\text {a }}$ Sesión realizada en el Principado de Mónaco, donde fueron aprobadas por unanimidad de los 96 miembros COI presentes. Si esta reforma se hubiera tramitado a través de un Congreso Olímpico, seguramente el resultado hubiese sido diferente.

Las recomendaciones fueron muy elegantemente empaquetadas, con el argumento de que para ver la pintura completa tenían que ensamblarse las 40 piezas como un rompecabezas, cerrando así, muy hábilmente, la posibilidad de un amplio debate que pudiera dejar por fuera algunas de ellas. Prueba de lo anterior es que, pese a las 83 intervenciones, algunas de ellas repetitivas, las deliberaciones previas a la aprobación, por unanimidad, de la Agenda solo tomaron la mitad del tiempo previsto para su discusión en el programa original de la Sesión. 
El rompecabezas gira en torno a tres ejes fundamentales: sostenibilidad, credibilidad y juventud. El eje de juventud es casi una constante en cualquier propuesta de reforma en cuanto los jóvenes son la esencia del Movimiento Olímpico. En cambio, los ejes de sostenibilidad y credibilidad son particulares a la actual coyuntura por la que atraviesa el deporte mundial, que tiene comprometidas a sus estructuras. La sostenibilidad es muy pertinente dadas las crecientes alternativas que se les presentan a los jóvenes hoy, en medio de la globalización y la era digital, representada en las redes sociales. La Credibilidad, por su parte, resultó ser premonitoria a los escándalos mundiales que, no solo desenmascararon a los poderosos dirigentes, sino a reconocidos atletas como el ciclista Lance Amstrong, los atletas Ben Johnson y Tyson Gay, los beisbolistas Barry Bonds y Alex Rodríguez, la atleta Marion Jones, el mismo Diego Maradona y más recientemente la tenista rusa Maria Sharapova.

\section{Una hoja de ruta para el futuro}

Dado que 40 es un número muy amplio de recomendaciones, lo que resulta poco práctico a la hora de recordarlas, éstas fueron distribuidas en los siguientes cinco grupos: 1) La particularidad de los Juegos Olímpicos (13 recomendaciones); 2) Los Atletas en el corazón del Movimiento Olímpico (5 recomendaciones); 3) Olimpismo en acción: mantener el Olimpismo vivo 365 días al año (8 recomendaciones); 4) El papel del Comité Olímpico Internacional: Unidad y Diversidad (10 recomendaciones); 5) La estructura y organización del COI (4 recomendaciones).

Las 40 recomendaciones no se listan, sino que aparecen inmersas en el texto, donde son reconocidas como (R. \#1), por ejemplo.

\section{La particularidad de los Juegos Olímpicos}

El primer grupo de recomendaciones de la Agenda Olímpica 2020 gira alrededor de los atletas, como era de esperarse, sino en torno a los Juegos Olímpicos. A pesar de que los atletas son la razón de ser del Movimiento Olímpico; sin atletas no habría Juegos Olímpico y sin estos, probablemente, no existiría el COI, es precisamente por lo anterior que las primeras 13 recomendaciones de la Agenda, buscan preservar la grandeza de los Juegos, garantizando así, la existencia de los atletas.

Sin duda, los Juegos Olímpicos, especialmente los de verano, son el evento más importante que celebra la humanidad, cada cuatro años; no solo por el número de países y territorios que logra convocar (en Río 2016 serán 206 países), sino por la cobertura de los medios de comunicación y la presencia en redes sociales. Los Juegos Olímpicos de Londres 2012 originaron más de 100.000 horas combinadas de transmisión, mientras que su página oficial registró más de 431 millones de visitas. (International Olympic Committee, 2013, p. 2)

Aun cuando algunos pueden argumentar que el Campeonato Mundial de Fútbol es el mayor evento, este no se puede comparar con unos Juegos Olímpicos por varias razones: a) en la fase final del mundial de fútbol sólo participan 36 países contra los 206 de los Juegos Olímpicos, b) la sede del mundial de fútbol se le otorga a un país que dispone múltiples subsedes, mientras que los Juegos Olímpicos se la adjudican a una sola ciudad, c) en un mundial de fútbol solo se juegan 64 partidos en un mes, mientras que en los JJ OO se disputan 310 pruebas en 16 días, d) el mundial de fútbol corona a un solo equipo campeón, mientras que los JJ OO entregan más de 300 medallas de oro a sus vencedores. Finalmente, podría argumentarse en favor de los Juegos Olímpicos que en estos participan, casi en igual proporción, mujeres y hombres. 
El objetivo principal de esta serie de recomendaciones es ampliar el número de ciudades del mundo elegibles para ser sede de unos Juegos Olímpicos; y para lograrlo propone una serie de recomendaciones muy concretas, empezando por la flexibilización y reducción de los costos de manejo de los Juegos ( $\mathrm{R}$ \#12), partiendo de la reducción de los costos de la candidatura (R. \#3). Para lograrlo se introduce una fase dentro del proceso de candidatura denominada de invitación ( R. \#1), donde conjuntamente la ciudad candidata y el COI evalúan las oportunidades y los riesgos ( R. \#2) de ser sede, priorizando aspectos como la sostenibilidad (R. \#4), no sólo ambiental, sino de mantenimiento de los escenarios deportivos, para lo cual se abre la posibilidad de que los Juegos se realicen en más de una ciudad, inclusive de un país y de que se usen escenarios deportivos temporales o desmontables.

Londres 2012 fue ya un buen ejemplo de estas prácticas sostenibles: 98\% de los materiales de las demoliciones fueron reciclados, se creó un micro hábitat de 45 hectáreas con un plan de manejo a 10 años para estimular la biodiversidad, se sembraron 300.000 plantas en el parque Olímpico, 62\% de los desperdicios de los Juegos fueron reciclados o convertidos en compost y el escenario de baloncesto fue totalmente desmontado después de los Juegos. (International Olympic Committee, 2013, p. 5)

Otras estrategias incluyen la reafirmación de los límites del marco para el Programa Olímpico (R. \#9), en términos de participación y de pruebas o eventos así: 10.500 atletas, 5.000 personas de apoyo y 310 pruebas para los Juegos de invierno y 2.900 atletas, 2.000 personas de apoyo y 100 pruebas para los de invierno. De otro lado se propone moverse de un programa basado en deportes a uno basado en eventos (R. \#10) y le da la posibilidad a la ciudad sede de incorporar nuevos eventos al programa; medida poco fácil de implementar sin que aumente en número de atletas participantes en los Juegos. Es preciso recordar que toda prueba o evento conducen a la disputa de las medallas y hace parte de una disciplina que, a su vez, conforma un deporte. Por ejemplo, la prueba de persecución individual hace parte de la disciplina de pista del deporte del ciclismo o el salto de plataforma de 10 metros hace parte de la disciplina de salto o clavados del deporte de la natación.

El COI ha sido consistente en combatir el gigantismo de los Juegos y lo ha reafirmado manteniendo el límite en el número de atletas, de deportes y de eventos, lo que obliga a depurar las disciplinas y los eventos, menos atractivos o muy parecidos. La inclusión de nuevos eventos propuestos por la ciudad sede, representa un desafío y eventual conflicto para las Federaciones Internacionales más poderosas con mayor número de eventos, para la televisión y sus ratings y para los patrocinadores del Movimiento Olímpico, las principales fuentes de ingresos del COI. La Agenda también promueve participación femenina hasta llegar al 50\% y la realización de más eventos mixtos.

Para los Juegos Olímpicos de 2020, donde se implementará por primera vez esta recomendación, el Comité Organizador de los Juegos Olímpicos de Tokio (COJOT) está proponiendo la inclusión de 18 eventos, entre ellos Béisbol masculino (deporte nacional en Japón) y Softbol femenino, deportes estos que fueron eliminados del programa de los Juegos Olímpicos después de Beijing 2008 para dar cabida el Golf y al Rugby 7 en Río 2016, decisión que obedece a los intereses de la televisión y los patrocinadores. ¿Cómo conciliar, entonces, la pretensión del COJOT 2020 de incluir el Béisbol y el Softbol con los poseedores de los derechos de transmisión de los Juegos? Los otros deportes propuestos son karate, monopatín, escalada deportiva y surfing. ¿Cuáles serán incluidos y cuáles serán eliminados? Esa respuesta la conoceremos en la próxima Sesión del COI a celebrarse en Río en agosto de este año.

Hay otro grupo de recomendaciones que están orientadas a fortalecer relaciones o "maximizar sinergias" con una serie de actores claves del Movimiento Olímpico como las mismas Federaciones Deportivas (R. \#13), ampliando su participación en la organización de los Juegos y 
trasladándoles la responsabilidad del manejo técnico, antes en cabeza de sus Comités Organizadores. También se invita a trabajar con las ligas profesionales (R. \#8), con el propósito de garantizar que los mejores atletas del mundo participen en los Juegos Olímpicos. No obstante, lo anterior, el fútbol continúa siendo una excepción a este deseo, al limitarse la edad de los jugadores a 23 años, con excepción de tres de ellos que no tienen límite de edad.

Justamente, una de las mayores incoherencias del programa de los Juegos Olímpicos, solo explicada por los intereses de la televisión y de los patrocinadores, es la forzada inclusión del fútbol el cual requiere, de una parte, habilitar varias subsedes, y de la otra, iniciar las competencias antes de la inauguración de los Juegos, pues 16 días no son suficientes para desarrollar el evento que implica la disputa de 6 partidos para los cuatro equipos que finalmente disputan las medallas; en ambos casos contraviniendo el espíritu de los Juegos. La misma Carta Olímpica, donde se codifican los Principios Fundamentales del Olimpismo, que rige el funcionamiento del Movimiento Olímpico y fija las condiciones de celebración de los Juegos Olímpicos, en su artículo 38 se refiere a la Villa Olímpica como el lugar de reunión de todos los atletas, oficiales y personal de apoyo (Comité Olímpico Internacional, 2015, p. 79), en la cual generalmente no están los futbolistas, quienes tampoco participan de las ceremonias de apertura y clausura de los Juegos. Si el COI realmente quiere rebajar costos en la organización de los Juegos Olímpicos debería considerar seriamente eliminar al fútbol del programa.

De acuerdo con su página web, el COI reconoce a 61 organizaciones Internacionales, lo que significa que eventualmente podrían ser beneficiarias de apoyos económicos por parte del COI. A pesar de lo anterior, la Agenda solo propone fortalecer las relaciones con algunas de ellas, específicamente la Asociación Internacional de los Juegos Mundiales -IWGA-, con la Asociación de Comités Olímpicos Nacionales -ACNO-, con diferentes escuelas de formación deportiva o "laboratorios del deporte", en las sedes de los Juegos Olímpicos, especialmente los de la Juventud (R. \#6) y con organizaciones que manejan el deporte para personas con diferentes habilidades (R. \#7), "apoyándolos con asistencia técnica y de promoción a través del Canal Olímpico". Resulta extraño que no se incluyan, dentro de aquellas con las que se busque fortalecer relaciones, a organizaciones tan importantes como el Comité Paralímpico Internacional, SportAccord, que agrupa a todas las federaciones deportivas internacionales Olímpicas y no Olímpicas, ni a ninguna organización relacionada con educación y diseminación del ideal olímpico, por ejemplo, la Academia Olímpica Internacional.

Al respecto, recientemente, el presidente de SportAccord, Marius Vizer, tuvo que renunciar a su cargo tras perder el apoyo de más de 20 Federaciones Internacionales afiliadas como resultado de su discurso pronunciado durante apertura de la Asamblea General realizada en Sochi en abril de 2015, donde criticó severamente al COI calificándolo de anticuado, injusto y poco trasparente. En su carta de renuncia Vizer expresó: "Me retiro con honor y por el honor del deporte" y agregó: "Hoy, el sistema que trabaja de puertas para adentro está dictado por los títulos nobiliarios o títulos familiares heredados, o por miembros designados de por vida; por lo que deseo que, en el futuro, los criterios básicos del sistema sean dictados por los logros deportivos, profesionalismo, desempeño, juego limpio, transparencia y el valor para expresar la verdad" (Play the Game, 2015).

Otras recomendaciones contenidas en el primer grupo, pero que poca relación guardan con los Juegos Olímpicos, buscan incluir la sostenibilidad en todos las operaciones diarias del Movimiento Olímpico (R. \#5), y promover la igualdad de género (R. \#11); frente a lo cual, ya se han implementado medidas para aumentar significativamente la participación femenina, no solo en los Juegos Olímpicos donde en Londres 2012 representaron el 44.2\% del total de atletas compitiendo, por primera vez, en todos los deportes tras la inclusión del boxeo femenino, sino en todos los ámbitos del Movimiento Olímpico. Actualmente de los 92 miembros activos del COI, 
22 son mujeres y 5 más son miembros honorarios, 4 de ellas forman parte del Comité Ejecutivo, $32 \%$ de los miembros de las nuevas comisiones del COI son mujeres, 11 mujeres son Presidentas de Comités Olímpicos Nacionales y 30 más son Secretarias Generales, y $26 \%$ de los Comités Ejecutivos de las Federaciones Internacionales están compuestos por mujeres (International Olympic Committee, 2016).

La posibilidad de celebrar los Juegos en diferentes ciudades, inclusive en diferentes países, debe ser la excepción a la regla en tanto que atentaría contra el verdadero espíritu de los mismos, amenazando con su disolución. Bien lo expresó durante la Sesión de aprobación de la Agenda, Denis Oswald, miembro COI de Suiza: "temo que el carácter único de los Juegos sea minimizado, la Villa Olímpica no funcione pues algunos atletas estarían aislados y no podrían ni siquiera asistir a la ceremonia de inauguración y el legado de los Juegos también se vería afectado" (International Olympic Committee, 2014).

En cuanto a la inclusión de nuevos deportes, no va a ser fácil definir la participación de las Federaciones Internacionales de los deportes admitidos. ¿Tendrán derecho a recibir dineros del COI sin ser parte del programa Olímpico? ¿Quién y con qué criterio define cuáles son los eventos que deberán ser excluidos de los Juegos para dar cabida a los admitidos? Al respecto, no hubo claridad durante la discusión de la Agenda, donde Franco Carraro, Miembro COI de Italia, portavoz del Grupo de trabajo 4, frente a una pregunta de Mikaela Cojuangco, miembro COI de Filipinas, aceptó la posibilidad de que se aumentara el número de deportes, siempre y cuando no aumente el número de eventos; con lo cual se ratifica la obligatoriedad de eliminar algunos eventos de otros deportes (International Olympic Committee, 2014).

\section{Los atletas en el corazón del Movimiento Olímpico}

A diferencia del último Congreso Olímpico celebrado en Copenhague en 2009, donde los atletas fueron el primer tema, ahora aparecen en el segundo grupo, reflejo de una nueva era donde los intereses comerciales priman sobre los de los atletas, a pesar de que se les pretenda ubicar en el corazón del Movimiento Olímpico. Las cinco recomendaciones de este capítulo giran en torno a la no discriminación, la exaltación de los "atletas limpios" y programas de apoyo para los atletas.

La primera acción de este grupo fue inmediata, contundente y muy liberal. En la misma Sesión se modificó y aprobó, por unanimidad, el sexto Principio Fundamental del Olimpismo (R. \#14), no solo para adaptarse a las actuales tendencias sociales, sino para evitar que fuese encasillado como un Movimiento retrogrado y conservador. Se trata de una medida incluyente y antidiscriminatoria donde se incorpora a la Carta Olímpica el rechazo a nuevas formas de discriminación basadas en "la orientación sexual, el idioma, las opiniones políticas, el origen nacional, la clase social y su condición económica". ¿Será lo anterior resultado de la convicción genuina de los miembros del COI, o una estrategia tendiente a atraer más simpatizantes al Movimiento?

La Agenda 2020 les declara la guerra frontal a dos de las más graves amenazas del deporte contemporáneo: el dopaje y la corrupción asociada al amaño de partidos y la manipulación de resultados, y lanza una cruzada para exaltar y proteger a los "atletas limpios" (R. \#15), estrategia que pretende quitarle el protagonismo a los atletas tramposos y, de paso, enviarle un fuerte mensaje para los medios de comunicación, especialmente aquellos de tendencia amarillista. Con esta iniciativa se busca, igualmente, recuperar el espíritu de los Juegos Olímpicos de la antigüedad donde:

"Los atletas ganadores eran considerados héroes en sus polis natales donde les erigían monumentos que perpetuaran su memoria y levantaban estatuas que primero fueron de madera, luego de bronce y de mármol colocadas en Olimpia o en su polis. [...] En los monumentos se grababa el nombre 
del vencedor, del padre, de la polis y del escultor. Las inscripciones eran, a veces en verso"

(Briceño Jáuregui, S.I., 1990, pág. 178).

Para combatir las amenazas, el COI destina cerca de USD \$20 millones (R. \#16), divididos en dos fondos de $\$ 10$ millones: uno, para luchar contra resultados viciados, manipulación y corrupción en el deporte y el otro para apoyar proyectos que ofrezcan nuevas aproximaciones científicas contra el dopaje.

Se honrará a los atletas limpios (R. \#17), que obtengan medallas Olímpicas como resultado de la descalificación de otros atletas por casos de dopaje, imponiéndoles sus medallas en ceremonias solemnes. Se queda corta esta bien intencionada iniciativa, en el sentido de que el escenario adecuado para desagraviar a estos atletas limpios es justamente en los propios Juegos Olímpicos, por lo que se debiera instaurar una ceremonia especial en cada edición, para entregar estas medallas, maximizando la importancia de la iniciativa.

Finalmente, el COI fortalece el apoyo a los atletas (R. \#18), poniendo a su disposición varios programas implementados a través de las plataformas tecnológicas que manejan los jóvenes de la generación de la Tecnología, la Información y las Comunicaciones, o era digital. El The Olympic Athletes' Hub (Central para Atletas Olímpicos) pone al servicio de los atletas una serie de herramientas que incluye cursos de formación, biblioteca virtual, información sobre la comisión de atletas, ofertas laborales y foros en redes sociales. Adicionalmente, hay un página denominada Compete, Learn \& Share (Compita, Aprenda y Comparta) dedicada a los Juegos Olímpicos de la Juventud (http://www.yogger.olympic.org) que como novedad, el Hub tiene un link con una "línea caliente" denominada Compliance and Integrity Hotline (Línea de cumplimiento e integridad) donde los atletas pueden, en forma anónima, denunciar cualquier sospecha relacionada con manipulación de competencias o violación al código de ética del COI, para lo cual le garantizan mantener la confidencialidad o simplemente se puede hacer la denuncia en forma anónima. (https://hub.olympic.org).

El Athletes Learning Gateway, (Entrada al Aprendizaje de los Atletas) también disponible para el público en general, es una excelente plataforma donde se ponen a disposición una serie cursos de cursos online y artículos publicados, cuyo propósito principal es potenciar al desempeño de los atletas durante la competencia y ayudarles a planificar su futuro después de su retiro como atletas activos. Actualmente están disponibles una serie de cursos sobre ciencias del deporte, entrenamiento deportivo, deporte y sociedad y el negocio del deporte que ofrecen capítulos como manejo del sueño, nutrición o manejo de la imagen del atleta. Tanto los cursos como los artículos disponibles son preparados por académicos de varias universidades universalmente reconocidas por atletas activos o retirados, quienes comparten sus experiencias en forma muy profesional. (http://onlinecourse.olympic.org).

\section{Olimpismo en acción: mantener el Olimpismo vivo 365 días al año}

Del título de este grupo de Recomendaciones se evidencia la necesidad de reorientar la misión del COI para que el Olimpismo juegue un papel más protagónico en forma permanentemente y no solo durante la celebración de las diferentes versiones de los Juegos Olímpicos; sin lo cual es prácticamente imposible fidelizar a una masa permanente de seguidores. Es necesario garantizar una interacción permanente con los diferentes actores y causas sociales alrededor del mundo; entre otras cosas, para estar a tono con esa nueva tendencia mundial conocida como Responsabilidad Social Empresarial.

Dentro de las ocho recomendaciones de este grupo, quizá la más significativa y la de mayor impacto de toda la Agenda Olímpica 2020, es la de lanzar el canal Olímpico (R. \#19), un 
viejo anhelo del COI que aquí se materializa. Su importancia se evidenció con el hecho de que Thomas Bach personalmente lideró este tema durante la etapa de formulación de la Agenda; y no es para menos, pues podría incrementar sustancialmente no solo los ingresos del COI, sino su imagen a través de la difusión permanente de las iniciativas y programas sociales a los cuales se encuentra vinculado. El Canal Olímpico representaría una oportunidad para los 206 Comités Olímpicos Nacionales, las 35 Federaciones Internacionales, los 12 Patrocinadores del programa TOP (The Olympic Partners), y en general para todas las partes interesadas de difundir sus programas y actividades durante los extensos períodos de tiempo entre Juegos Olímpicos. El canal Olímpico será, exclusivamente digital, disponible a través de los diferentes dispositivos móviles, los cuales están, en su mayoría en poder de la nueva generación de jóvenes a quienes más se les facilita su uso.

Durante los 16 días de los Juegos Olímpicos de Londres, los seguidores del COI a través de todas sus redes sociales aumentaron en 4.1 millones, alcanzando los 20 millones de fans. (International Olympic Committee, 2013), una no despreciable base que justifica una interacción permanente como vehículo de comunicación para difundir la misión y la visión del COI a todo el mundo. Desde el Congreso Olímpico de Copenhague en 2009, el COI se lanzó a la conquista de las redes sociales, entendiendo que su mercado potencial está entre los jóvenes, a quienes "hay que hablarles en su propio idioma".

Al respecto, Sir Martin Sorrell, muy reconocido hombre de negocios inglés, en el campo de las comunicaciones, ha sido un inspirador determinante para el COI a través de sus intervenciones en el Congreso Olímpico de Copenhague y como conferencista invitado a la $128^{\mathrm{a}}$ Sesión del COI realizada en Kuala Lumpur en 2015. Frente a mi pregunta, "el canal Olímpico del COI [en YouTube] tiene únicamente 19,982 suscriptores y 754,528 vistas; por ejemplo, su fantástico discurso, en este momento tiene 50 vistas y solo un like, el mío, lo cual es muy poco comparado con otros canales, inclusive de deportes como el de FIFA (1.202.980 suscriptores y 211.140.303 vistas); ¿Significa esto que el mensaje Olímpico no le está llegando a los jóvenes?”, Sorrell respondió: "En cuanto al COI, yo espero que la audiencia crezca; ya lo veremos. FIFA probablemente atrae, por la razón equivocada" Y agregó: Tengo gran fe en el Presidente Bach y su fuerte liderazgo..." (Sorrell, 2015).

La razón equivocada en la FIFA a la que se refiere Sir Martin tiene que ver con el hecho de que los escándalos atraen audiencias millonarias, lo cual explica, pero al mismo tiempo dificulta la buena intención del COI de cambiar el foco de atención hacía los "atletas limpios". Para lograr el cambio se requiere de los medios de comunicación, los cuales lamentablemente están regidos por el rating, el cual se dispara ante los grandes escándalos.

Fortalecer la capacidad de influencia del COI (R. \#21) y establecer alianzas estratégicas (R. \#20) parecen hacer parte de la nueva misión del COI, en reemplazo de algunas políticas asistencialistas como las aplicadas en programas como "Sport for Hope" (Deporte para la esperanza) (R. \#24), que han resultado en cuantiosas inversiones por parte del COI, sin un retorno acorde en Haití y Zambia. Otras recomendaciones de este grupo están dirigidas a los programas sociales que promueve el COI, la mayoría con poco éxito como es el caso del programa de Educación Olímpica basada en Valores -OVEP- (R. \#22) y el compromiso con comunidades de jóvenes, voluntarios y público en general, además de los atletas.

El Programa OVEP es el resultado del trabajo de la antigua comisión de Cultura y Educación Olímpica (ahora Comisión de Educación Olímpica) del COI. El objetivo de OVEP es descubrir los valores asociados al deporte e incorporarlos a los procesos educativos de los niños y jóvenes, a través de sus docentes y entrenadores deportivos. Para contribuir con este propósito se ha diseñado un manual de enseñanza que sirve como referencia para profesores y educadores dispuestos a promover los valores del Olimpismo; invitándolos a usar los símbolos y las historias 
Olímpicas como parte de un programa de estimulación interactiva. En la presentación del programa, el entonces presidente del COI Jacques Rogge indicó: "Nuestro mundo hoy necesita paz, tolerancia, y hermandad. Combinando deporte con cultura y educación, los valores Olímpicos pueden contribuir a alcanzar este propósito. [...] El deporte trae esperanza, orgullo, sentido de pertenencia y salud, moldeando así el cuerpo y el alma." (International Olimpic Committee, 2007, p. 7).

Tanto Sport for Hope como OVEP son programas que hacen parte de la estrategia global del COI para promover entre los jóvenes los valores Olímpicos a través del deporte. Sin embargo, como bien lo reconoce el COI, Sport for Hope está en evaluación; mientras que OVEP está aún en curso a pesar de haberse lanzado en 2007; pues hasta ahora, solo 17 iniciativas han sido presentadas en todo el mundo.

Una de las más sorprendentes recomendaciones de la Agenda es la de "Revisar el posicionamiento de los Juegos Olímpicos de la Juventud" (R. \#25), no porque no deba ser revisada, sino porque es uno de los más importantes legados del antecesor de Bach, el belga Jacques Rogge, de quien fuera su vicepresidente por 10 años. A pesar de tener muchas voces en contra, los Juegos Olímpicos de la Juventud, tantos de verano como de invierno, representan una serie de oportunidades para los atletas que participan como para las ciudades que los realizan y sirve como un laboratorio para experimentar con diversas iniciativas antes de introducirlas en los juegos "mayores", tales como inclusión de disciplinas mixtas, e inclusive, la conformación de equipos integrados por varios países.

Con la recomendación de combinar aún más deporte y cultura (R. \#26), se busca mantener viva la idea de Pierre de Coubertin, conocido como el instaurador de los Juegos Olímpicos de la era moderna, pero sobre todo era un educador reformista francés, quien creía firmemente en que el deporte contribuía al desarrollo armonioso y equilibrado del cuerpo, el carácter y la mente. Combinar arte y deporte Olímpico no era una idea nueva de Coubertin, sino una vieja tradición qué él mismo buscaba ayudar a restablecer (Guillain, 2009, p. 148).

El ideal Olímpico de vincular deporte y cultura ha estado asociado a los Juegos Olímpicos desde tiempos inmemoriales hasta nuestros días, especialmente en tres momentos históricos: Primero, durante los Juegos Olímpicos de la Antigüedad en los cuales, a través de diferentes expresiones artísticas, se honraba e inmortalizaba a los atletas vencedores; segundo, durante los Juegos Olímpicos de la era moderna, en la primera mitad del siglo XX, donde competencias de arquitectura, escultura, pintura, música y literatura hicieron parte del programa oficial de los Juegos; tercero, a partir del año 2000, en el que la Comisión de Cultura y Educación Olímpica del COI estableció Competencias de deporte y arte Olímpico en categorías de escultura y artes gráficas, realizando convocatorias preliminares a través de los Comités Olímpicos Nacionales. (Villegas Estrada, 2014). La Agenda 2020 busca fortalecer este vínculo, más allá de los Juegos Olímpicos, a través de las siguientes estrategias: 1) crear el premio "Laurel Olímpico" para destacar contribuciones excepcionales al Olimpismo en cultura, educación, desarrollo y paz, 2) crear una Casa Olímpica, 3) construir un Museo Olímpico itinerante y 4) designar un "agregado" de la Cultura Olímpica por parte de los Comités Olímpicos Nacionales.

\section{El papel del Comité Olímpico Internacional: Unidad y Diversidad}

Este cuarto grupo, que aglutina diez recomendaciones, a pesar de no contener las reformas más profundas o novedosas, tiene como propósito responder a la reciente crisis de confianza en que está inmerso el deporte mundial. Los escándalos de corrupción y las sospechas de amaño de partidos y manipulación de resultados y casos de resultados analíticos adversos en dopaje han 
encendido las alarmas en el COI, frente a lo cual propone implementar una serie de acciones tendientes a restaurar la credibilidad y la imagen del deporte.

Para contrarrestar la mala imagen y evitar que la situación se salga de control, la Agenda 2020 lanza una cruzada para aumentar la transparencia (R. \#29) y mejorar las prácticas de las organizaciones deportivas, acogiéndose a una serie de principios básicos, universales, de buena gobernanza (R. \#27). A pesar de las buenas intenciones, no es fácil concertar unos principios universales dada la gran diversidad de culturas, ideologías y valores aceptados en cada sociedad. No necesariamente lo que es aprobado, malo o prohibido en una cultura lo es en otra.

Para facilitar el consenso en torno a los principios básicos universales, el COI está empeñado en respaldar la autonomía del Movimiento Olímpico frente a las autoridades nacionales (R. \#28); para lo cual ha propuesto crear un modelo de memorando de entendimiento estándar para ser suscrito con los gobiernos nacionales. En este sentido, el COI también ha mantenido una política consistente, llegando al extremo de desafiliar temporalmente a Comités Olímpicos Nacionales que han sido intervenidos por los Gobiernos, o que no han permitido la libre movilidad de los deportistas. El más reciente caso tiene que ver con el Comité Olímpico de Kuwait, suspendido el pasado 27 de octubre de 2015, por negarse a derogar una modificación a la Ley del deporte en la que el Gobierno pretende interferir con el Movimiento Olímpico. Paradójicamente, el Sheikh de Kuwait Ahmad Al-Fahad Al-Sabah, uno de los más influyentes miembros del COI, es actualmente el Presidente de la Asociación de Comités Olímpicos Nacionales (ANOC) y de la Comisión de Solidaridad Olímpica, que entrega las asistencias financieras a los Comités Olímpicos Nacionales.

Otra medida impartida es el fortalecimiento de la independencia de la Comisión de Ética (R. \#30), cuyos miembros ya no son elegidos por el Comité Ejecutivo del COI, sino por todos sus miembros en la Sesión, mediante votación secreta. Adicionalmente, se crea un nuevo cargo dentro de la estructura del COI, denominado Oficial de Ética y Cumplimiento (R. \#31), para el cual fue nombrada la señora Pâquerette Girard Zappelli

Un significativo logro y, de paso muestra de eficiencia de la Comisión de Ética fue el fortalecimiento de la ética (R. \#32) materializado en la formulación del nuevo Código de Ética del COI, ampliado significativamente para adecuarse a la nueva realidad del deporte mundial. Resultado de lo anterior, recientemente se lanzó la edición 2016 de la colección de Ética del COI, compuesta, además de los nuevos estatutos de la Comisión por: a) Un nuevo Código de Ética y sus normas de aplicación que incluyen las reglas de conducta para el proceso de candidatura de los Juegos de 2024 y el reglamento de los conflictos de interés; b) la Declaración de Principios Básicos de Buena Gobernanza del Movimiento Olímpico y el deporte en general y c) el nuevo Código de Prevención de Manipulación de Competiciones y sus normas de aplicación para los Juegos de Rio.

Como resultado de las recomendaciones relacionadas con la ética, se diseñó e implementó la ya mencionada línea especial de denuncias (Integrity and Compliance Hotline) que busca contribuir a la globalización de la lucha contra la corrupción. Se trata de un mecanismo para denunciar potenciales casos de manipulación de partidos, así como otras violaciones al Código de Ética del COI, a través de la plataforma del COI que le permite al denunciante mantenerse en el anonimato a la vez que la información recibida se maneja con confidencialidad.

Por otra parte, dentro del grupo dedicado al papel de COI, se efectuaron cuatro recomendaciones relacionadas con mercadeo y merchandising cuyo propósito fundamental es activar al Club de los socios del programa TOP para que se involucren directamente en algunos programas sociales del COI, maximizando así el valor de sus marcas y dándole un enfoque más social. Componen el Programa TOP: Coca Cola (desde 1928), Atos SE, Bridgestone, Dow 
Chemical, General Electric, McDonalds, Omega, Panasonic, Proctel \& Gamble, Samsung, Toyota y Visa. Una estrategia es involucrar a los patrocinadores con el programa Olimpismo en Acción (R. \#33) para que contribuyan a su financiación, difusión y aplicación. Para fortalecer el compromiso de los Comités Olímpicos Nacionales -CONs- con los patrocinadores del programa TOP (R. \#35), se reunieron en Lausana con el propósito de identificar nuevas "oportunidades de negocio" que, ojalá no solo sirvan para aumentar los ingresos del COI, sino para ampliar sus incipientes programas sociales.

De otro lado, el COI busca desarrollar un programa global de licencias (R. \#34) más orientado a la promoción de la marca Olímpica que a la generación de ingresos; interesante estrategia para acercar el Movimiento Olímpico a las personas comunes y corrientes, de tal manera que un niño pueda llevar a casa una mascota oficial de los Juegos sin dejar, a cambio, 50 euros en las nutridas arcas del COI. Adicionalmente, se pretende extender el acceso a la marca olímpica para uso no comercial (R. \#36); es decir, que se le permitirá su uso a Organizaciones no comerciales que contribuyan al Movimiento Olímpico y a los Juegos.

\section{La estructura y organización del COI}

El último grupo de la Agenda Olímpica 2020 es a su vez el menos profundo; no sólo tiene el menor número de recomendaciones, sino que no presenta ningún cambio significativo en la estructura y organización del COI. Entre las medidas que se propone adoptar está evaluar la edad límite de los miembros del COI (R. \#37). La actual edad límite para los miembros del COI electos después de 1999 es de 70 años, para los nombrados entre 1967 y 1999 es de 80 años y no tienen edad límite quienes ingresaron al COI antes de 1966, siendo los dos únicos sobrevivientes el Grand Duque Jean de Luxemburgo quien ingresó en 1946, a los 25 años de edad; y el Rey Constantino de Grecia quien ingresó 1963, con tan solo 23 años de edad. Las reducciones en la edad límite de los miembros COI van en contravía de las leyes de la naturaleza. Entre mayor es la expectativa de vida de las personas, menor es el límite de edad de los miembros COI.

Resultado de las políticas del COI sobre la edad límite de sus miembros, se corre el riesgo de perder personas valiosas y útiles para el Movimiento Olímpico. Un ejemplo de lo anterior es Andrés Botero Phillipsbourne, quien a pesar de haberse coronado campeón mundial de motonáutica y ejercer con plenitud de sus facultades físicas y mentales el cargo de Ministro del Deporte en Colombia, fue retirado del COI, por haber cumplido la edad límite de 70 años, sin alcanzar, siquiera a la membrecía de Honor, por sólo haber servido ocho años al Movimiento, dos menos de los requeridos para tal honor.

No es la edad la que debe limitarse, sino el período o tiempo de servicio, el cual no debería sobrepasar los doce años, tiempo más que suficiente para contribuir significativamente al COI y dejar su legado.

A través de la historia, el COI ha estado estrechamente relacionado con las monarquías y la realeza. Actualmente son miembros COI el Príncipe Alberto II de Mónaco (1985), el Sheikh Al-Sabah de Kuwait (1992), la Princesa Real de Inglaterra (1988), la Infanta Doña Pilar de Borbón de España (1996), el Príncipe Al Hussein de Jordania (2010), el Sheick Al-Thani de Qatar (2002), el Barón Beckers-Vieujant de Bélgica (2012), el Príncipe Frederik de Dinamarca (2009), la Princesa Nora de Liechtenstein (1984), el Gran Duque de Luxemburgo (1998), el Príncipe Abdulazir Al de Arabia Saudita (2002), el Rey Willem-Alexander de Holanda (1998).

Una de las más importantes recomendaciones de la Agenda es la de implementar un proceso de selección dirigida para los miembros del COI (R. \#38), una interesante estrategia con la que se pretende alcanzar una mayor apertura del COI permitiendo la vinculación de nuevos 
miembros, escogidos por sus propios méritos, más que por sus títulos nobiliarios o capacidad de influencia. Resulta curioso, sin embargo, que esta medida no ha empezado a ser implementada, a pesar del anuncio, por parte de la presidenta de la nueva Comisión de Elección de Miembros COI, su Majestad la Princesa Ana de Inglaterra, de la difusión del nuevo protocolo de selección el cual fue prometido para finales del año 2015. Como resultado de lo anterior, en el momento existen 23 vacantes de miembros COI.

La actual estructura del COI es la de un club privado, gobernado por una serie de "socios" denominados Miembros COI, que se eligen y reeligen unos a otros, toman todas las decisiones trascendentales del Movimiento Olímpico y la mayoría de ellos no representan a nadie. Desde 1999 el número de "socios del club" ha sido limitado a 115 miembros: 70 de ellos son miembros individuales provenientes de igual número de países, 15 son atletas activos, otros 15 representan a las 35 Federaciones Internacionales ( 28 de verano y 7 de invierno) y los restantes 15 representan a los 206 Comités Olímpicos Nacionales.

La estructura vigente del COI tiene que cambiar, tiene que abrirse, tiene que pasar de ser ese club privado a, por lo menos, uno semiprivado, con algún grado de democracia. El COI tan solo "reconoce" a los CONs, los cuales no tienen ninguna afiliación con el COI. Los CONs no participan de las Sesiones ordinarias y extraordinarias del COI, lo que es un desacierto; los Presidentes de CONs, elegidos por sus Federaciones Nacionales, por derecho propio, deberían ser los Miembros del COI.

Al respecto, preocupa la inminente disminución de la representación iberoamericana en el seno del COI con la reciente salida de varios miembros activos como Melitón Sánchez de Panamá, Carlos Nuzman de Brasil y Julio Maglione de Uruguay (pasaron a ser honorarios), Andrés Botero de Colombia (retirado) y Reynaldo González de Cuba (fallecido), quienes serían reemplazados por nuevos miembros que seguramente provengan especialmente de África y Oceanía.

Otra interesante iniciativa es la de fomentar el diálogo con la sociedad y dentro del mismo Movimiento Olímpico (R. \#39), para lo cual se creó el “Congreso Olímpico en acción” otra medida para acercar el Movimiento a la gente. Justamente, el primero de estos Congresos se celebrará en Lima, Perú en 2017, como parte de las actividades de la $130^{\text {a }}$ Sesión del COI, en la cual, entre otras cosas, se definirá la sede de los Juegos Olímpicos de verano del año 2024.

Por último, la estructura y organización del COI ha sufrido un cambio extremo con la revisión del alcance y composición de sus Comisiones de trabajo (R. \#40). En la nueva composición de las 26 comisiones, se destaca la intención de darle un enfoque más social, de mayor cercanía a la gente del común.

Se eliminaron comisiones (Derechos de televisión y otros medios), se dividieron unas (Cultura y Educación), se fusionaron otras, se crearon nuevas (de Canal Olímpico y de Comunicaciones) y se trasformaron varias (ej. de Sostenibilidad y Legado antes de Deporte y Medio Ambiente; Médica y Científica antes Médica; del Entorno del Atleta antes de Entorno; de Deporte y Sociedad Activa antes de Deporte para Todos; de Elección de Miembros COI antes de Nominaciones; de Mujeres en el Deporte antes de Mujer y Deporte; de Asuntos legales antes Comisiones Jurídica y Deporte y Leyes; de Comisiones de Educación Olímpica y de Cultura y Patrimonio Olímpico antes de Cultura y Educación; de Asuntos Públicos y Desarrollo Social antes de Relaciones Internacionales). Finalmente, se mantuvieron las siguientes comisiones: de Prensa, de Radio y Televisión, de Atletas, Comité Auditor, de Coordinación, de Miembros Delegados, de Ética, Financiera, del Comité Ejecutivo, de Representantes en el Comité Ejecutivo de WADA, de Mercadeo, Comisión (Junta Directiva) de Sistema de Radiodifusión Olímpica, de Programa Olímpico, y de Solidaridad Olímpica. En total son 20 comisiones. 
11. Conclusiones

Aun cuando en el papel la Agenda Olímpica 2020, la estratégica hoja de ruta para el Movimiento Olímpico, propone sugestivas novedades, solo el tiempo y la historia se encargarán de mostrar que tan efectivas y profundas fueron las 40 recomendaciones. Las reformas propuestas son ilusorias para las candidaturas, generosas con los atletas, vitales para la sostenibilidad, refrescantes para el papel del COI y muy tenues frente a su estructura. Lamentablemente, la Agenda no propone indicadores que permitan evaluar la gestión y medir el impacto de las recomendaciones.

Es indiscutible que la iniciativa es audaz, por el momento en el que es presentada, por la metodología utilizada y por el liderazgo de su impulsor. Thomas Bach, logró que 15 meses después de su posesión, sin ninguna oposición, sus colegas miembros COI, le firmaran un cheque en blanco para emprender su camino reformista, el cual está recorriendo a gran velocidad pese el desconocimiento de la mayoría de sus partes interesadas. ¿Quiénes de los militantes Iberoamericanos del Olimpismo conocen el contenido de la Agenda? ¿Cuantas páginas web de Comités Olímpicos Nacionales o Federaciones Deportivas la incluyen en sus contenidos?

La flexibilización propuesta para el proceso de candidatura y organización de los Juegos Olímpicos no ampliará significativamente el círculo de ciudades candidatas y por el contrario atenta contra el verdadero espíritu de los Juegos en cuanto la sede le sea otorgada a más de una ciudad o país. De otro lado, el pasar de un programa basado en deportes a uno de eventos, sin la posibilidad de incrementar el número de atletas, generará conflictos entre las Federaciones Internacionales. La Agenda desconoce la realidad de los Juegos Olímpicos desde la experiencia de los aficionados del común, quienes cada vez tienen menos acceso por efecto de las políticas del COI para el manejo de la boletería, los excesivos costos, y la especulación asociada.

La intención de poner a los atletas en el corazón del Movimiento Olímpico, como corresponde, es uno de los aspectos más valiosos de la Agenda, a pesar de no ser una idea original en tanto ya se había incluido en las recomendaciones del último Congreso Olímpico realizado en Copenhague en 2009. Lo novedoso está en las herramientas tecnológicas puestas al servicio de los atletas y el propósito permanente de honrar a los "atletas limpios".

Se percibe a lo largo de la Agenda una intención de hacer más sociable al COI, más cercano a la gente y a otras organizaciones, vinculado con programas de responsabilidad social, La creación del Canal Olímpico es quizá la recomendación más importante de la Agenda por las oportunidades que representa, para todas las partes interesadas, de mantener vivo el Movimiento en los períodos entre Juegos.

La agenda pretende devolverle la relevancia a aspectos que no están cumpliendo su propósito, como el vínculo entre Olimpismo, Cultura y Educación. Las recomendaciones de crear el Congreso Olimpismo en Acción, de designar un "agregado de la Cultura Olímpica" en cada CON, de desarrollar una Casa Olímpica, de organizar un Museo Itinerante; y de crear el premio Laurel Olímpico para resaltar contribuciones excepcionales al Olimpismo en materia de cultura, educación, desarrollo y paz, son una muestra de las buenas intenciones del COI en esta materia.

Donde más corta se queda la Agenda es en el último grupo de recomendaciones, pues solo propone unos ajustes menores en la organización y, a pesar del cambio en el sistema de selección de los miembros COI, no se atreve a plantear una reforma profunda a su envejecida estructura. 
Desgranando las recomendaciones, rápidamente se evidencia que no todas tienen el mismo grado de importancia, contrario a lo expresado por Bach. No tiene el mismo impacto crear un Canal Olímpico ( $\# 19$ ), que garantizar la sostenibilidad en todas las operaciones diarias del COI (R \#5). De otro lado, hay recomendaciones cuya implementación tomará varios años como la de incluir la sostenibilidad en todos los aspectos de los Juegos Olímpicos (R \#4), mientras que otras quedaron implementadas, inclusive antes de concluir las deliberaciones de la Sesión donde fue aprobada la Agenda, tal y como ocurrió con la modificación del $6^{\circ}$ Principio Fundamental del Olimpismo (R \#14). En la parte puramente formal, pareciera que algunas recomendaciones estuvieran mal ubicadas dentro del "rompecabezas".

Tampoco es igual el grado de proactividad de las diferentes comisiones responsables de implementar las recomendaciones. Contrasta, por ejemplo, la diligencia de la Comisión de Ética que en muy poco tiempo sacó la colección de ética 2016 compuesta por varios documentos, mientras que la Comisión de Elección de los Miembros COI, aún no presenta el protocolo para la elección de nuevos miembros COI a pesar del compromiso de darlo a conocer antes de finalizar el 2015 .

\section{Bibliografía}

- Bogotá, D.C., Colombia: Imprenta Patriótica del Instituto Caro y Cuervo.

- Butler, N. (2016, february 8). inside the games. Recuperado el 17 de abril de 2016 de insidethegames.biz.

- Comité Olímpico Internacional. (2015). Carta Olímpica. Lausanna: Comité Olímpico Internacional.

- Elias, N., \& Dunning, E. (1995). Deporte y ocio en el proceso de la civilización. (P. Jimenez, Trans.) México, D.F., México: Fondo de Cultura Economica.

- Guillain, J.-Y. (2009). The End of the Olympic Art Contest. When art and sport became strangers to each other. (S. Wassong, Ed.) Journal of Olympic History, 148-154.

- International Olimpic Committee. (2007). Teaching Values, an Olympic Education Toolkit. (D. L. Binder, Ed.) Lausanne, Switzerland: IOC Commission for Culture \& Olympic Education.

- International Olympic Committee. (2013). London 2012 Facts and Figures. Factsheet, Lausanne.

- International Olympic Committee. (2014). Olympic Agenda 2010, Context and background. Lausanne: IOC.

- International Olympic Committee. (2014). Speech by Thomas Bach on the occasion of the Opening Ceremony 127th IOC Session. Olympic Agenda 2020 Context and background (p. 106). Monaco: International Olympic Committee.

- International Olympic Committee. (2014, december 8). Day 1 - Morning Session - Part 1 127th IOC Session in Monaco. Monte Carlo, Monaco.

- International Olympic Committee. (2015). IOC Annual Report 2014 Credibility, Sustainability and Youth. International Olympic Committee, Château de Vidy, 1007 Lausanne,. Lausanne: International Olympic Committee. 
- International Olympic Committee. (2016). Women in the Olympic Movement. Lausanne: IOC.

- Miller, D. (2008). The Official History of the Olympic Games and the IOC: Athens to Beijing, 1894-2008. Great Britain: Mainstream Publishing.

- Müller, N. (1994). One hundred years of Olympic Congresses. Laussane, Suiza: International Olympic Committee.

- Play the Game. (1 de junio, 2015). http://www.playthegame.org. Consultado el 26 de junio de, 2016.

- Sorrell, M. (8 de marzo, 2015). Your speech at the IOC Session - You did it again!. (C. E. Villegas, Interviewer, \& C. E. Villegas, Translator)

- Transparency International. (2016). Global Corruption Report: Sport. London: Routledge Taylor and Francis Group.

- Villegas Estrada, C. E. (2010). Recomendaciones del XIII Congreso Olímpico. Fundación Ayuda al Deporte. Bogotá: Fundación Ayuda al Deporte.

- Villegas Estrada, C. E. (2010). VII Congreso Mundial de Deporte, Educación y Cultura. Asistencia, Comité Olímpico Colombiano, Durban.

- Villegas Estrada, C. E. (26 de marzo, 2014). Olimpismo, cultura y educación, combinando deporte y arte, músculo y mente.Bogotá. Trabajo presentado en la Academia Olímpica de Colombia, Bogotá. Recuperado de:

https://issuu.com/carloseduardovillegase/docs/aoc_olimpismo_cultura_y_educacion_x 